\title{
THE RESTRICTED BURNSIDE PROBLEM FOR MOUFANG LOOPS
}

\author{
ALEXANDER GRISHKOV $^{\mathrm{A}}$, LIUDMILA SABININA ${ }^{\mathrm{B}, 1}$, EFIM ZELMANOV $^{\mathrm{C}}$ \\ To the memory of Peter Plaumann.
}

\begin{abstract}
We prove that for positive integers $m \geq 1, n \geq 1$ and a prime number $p \neq 2,3$ there are finitely many finite $m$-generated Moufang loops of exponent $p^{n}$.
\end{abstract}

\section{INTRODUCTION}

A loop $U$ is called a Moufang loop if it satisfies the following identities:

$$
((z x) y) x=z((x y) x) \text { and } x(y(x z))=(x(y x)) z
$$

In this paper we solve the Restricted Burnside Problem for Moufang loops of exponent $p^{n}, p>3$.

Theorem 1. For an arbitrary prime power $p^{n}, p>3$, there exists a function $f(m)$ such that any finite $m$-generated Moufang loop of exponent $p^{n}$ has order $<f(m)$.

For groups this assertion was proved by E. Zelmanov ([20], [21]). For Moufang loops of prime exponent it was proved by A. Grishkov [6] (if $p \neq 3$ ) and G. Nagy [15] (if $p=3$ ). In [16], [17] the Restricted Burnside Problem was solved for a subclass of Moufang loops and related Bruck loops.

2000 Mathematics Subject Classification. Primary 20N05, Secondary 17D10.

Key words and phrases. Moufang loop, Burnside problem, Malcev algebra. 


\section{Groups With triality}

A group $G$ with automorphisms $\rho$ and $\sigma$ is called a group with triality if $\rho^{3}=\sigma^{2}=(\rho \sigma)^{2}=1$ and

$$
[x, \sigma][x, \sigma]^{\rho}[x, \sigma]^{\rho^{2}}=1
$$

for every $x \in G$, where $[x, \sigma]=x^{-1} x^{\sigma}$.

Let $G$ be a group with triality. Let $U=\{[x, \sigma] \mid x \in G\}$. Then the subset $U$ endowed with the multiplication

$$
a \cdot b=\left(a^{-1}\right)^{\rho} b\left(a^{-1}\right)^{\rho^{2}} ; a, b \in U
$$

becomes a Moufang loop.

Every Moufang loop $U$ can be obtained in this way from a suitable group with triality, which is finite if $U$ is finite. Moreover, if $p$ is a prime number, then a finite Moufang $p$-loop can be obtained from a finite $p$-group with triality ([3], [5], [10]).

\section{Lie And MalCEV ALGEBras}

Let $\mathbb{F}_{p}$ be a field of order $p$, let $G$ be a group. Consider the group algebra $\mathbb{F}_{p} G$ and its fundamental ideal $\omega$, spanned by all elements $1-g, g \in G$. The Zassenhaus filtration is the descending chain of subgroups

$$
G=G_{1}>G_{2}>\cdots
$$

where $G_{i}=\left\{g \in G \mid 1-g \in \omega^{i}\right\}$. Then $\left[G_{i}, G_{j}\right] \subseteq G_{i+j}$ and each factor $G_{i} / G_{i+1}$ is an elementary abelian p-group. Hence,

$$
L=L_{p}(G)=\sum_{i \geq 1} L_{i}, \quad L_{i}=G_{i} / G_{i+1}
$$

is a vector space over $\mathbb{F}_{p}$. The bracket

$$
\left[x_{i} G_{i+1}, y_{j} G_{j+1}\right]=\left[x_{i}, y_{j}\right] G_{i+j+1} ; \quad x_{i} \in G_{i}, y_{j} \in G_{j},
$$

makes $L$ a Lie algebra. Notice that the bracket [, ] on the left hand side of the last equality is a Lie bracket whereas $[$,$] on the right hand$ side denotes the group commutator.

Let $x, y$ be generators of a free associative algebra over $\mathbb{F}_{p}$. Then $(x+y)^{p}=x^{p}+y^{p}+\{x, y\}$, where $\{x, y\}$ is a Lie element. Following 
[12, we call a Lie $\mathbb{F}_{p}$-algebra $L$ with an operation $a \rightarrow a^{[p]}, a \in L$, a Lie $p$-algebra if

$$
\begin{aligned}
(k a)^{[p]} & =k^{p} a^{[p]}, \\
(a+b)^{[p]} & =a^{[p]}+b^{[p]}+\{a, b\}, \\
{\left[a^{[p]}, b\right] } & =[\underbrace{a,[a, \ldots[a}_{p}, b] \ldots]
\end{aligned}
$$

for arbitrary $k \in \mathbb{F}_{p} ; a, b \in L$. The mapping $L_{i} \rightarrow L_{i p}$, $\left(g_{i} G_{i+1}\right)^{[p]}=g_{i}^{p} G_{i p+1}$, extends to the operation $a \rightarrow a^{[p]}, a \in L$, making $L$ a Lie $p$-algebra. For more details about this construction see [2], [1], [22].

We call a Lie algebra (resp. Lie $p$-algebra) $L$ with automorphisms $\rho, \sigma$ a Lie algebra with triality if $\rho^{3}=\sigma^{2}=(\rho \sigma)^{2}=1$ and for an arbitrary element $x \in L$ we have

$$
\left(x^{\sigma}-x\right)+\left(x^{\sigma}-x\right)^{\rho}+\left(x^{\sigma}-x\right)^{\rho^{2}}=0 .
$$

Lemma 3.1. Let $G$ be a group with triality and let $p$ be a prime number. Then $L_{p}(G)$ is a Lie p-algebra with triality.

Proof. The automorphisms $\rho, \sigma$ of the group $G$ give rise to automorphisms $\rho, \sigma$ of the Lie algebra $L_{p}(G)$. For an element $x_{i} \in G_{i}$ we have

$$
\left[x_{i}, \sigma\right]\left[x_{i}, \sigma\right]^{\rho}\left[x_{i}, \sigma\right]^{\rho^{2}}=1 .
$$

It implies that for the element $x=x_{i} G_{i+1} \in L_{i}$ we have

$$
\left(x^{\sigma}-x\right)+\left(x^{\sigma}-x\right)^{\rho}+\left(x^{\sigma}-x\right)^{\rho^{2}}=0 .
$$

This completes the proof of the lemma.

Recall that a (nonassociative) algebra is called a Malcev algebra if it satisfies the identities

(1) $x y=-y x$

(2) $(x y)(x z)=((x y) z) x+((y z) x) x+((z x) x) y$,

see [4], [14], [23].

Lemma 3.2 (see [7]). Let $L$ be a Lie algebra with triality over a field of characteristic $\neq 2,3$. Let $H=\left\{x \in L \mid x^{\sigma}=-x\right\}$. Then $H$ is a 
Malcev algebra with multiplication

$$
a * b=\left[a+2 a^{\rho}, b\right]=\left[a^{\alpha}, b\right],
$$

where $a, b \in H, \alpha=1+2 \rho$.

Lemma 3.3. For arbitrary elements $a, b, c \in H$ we have

$$
3[[a, b], c]=2(a * b) * c+(c * b) * a+(a * c) * b .
$$

We remark that in a Lie algebra with triality over a field $F$, for arbitrary elements $a_{1}, \ldots, a_{n} \in H$ the subspace $\sum_{i=1}^{n} F a_{i}+\sum_{i=1}^{n} F a_{i}^{\alpha}=$ $\sum_{i=1}^{n} F a_{i}+\sum_{i=1}^{n} F a_{i}^{\rho}$ is invariant with respect to the group of automorphisms $\langle\sigma, \rho\rangle$.

Proof. Let's prove that for any $x, y, z \in H$ :

$$
(x * y) * z=2\left[\left[x^{\rho^{2}}, y^{\rho}\right], z\right]+[[x, y], z] .
$$

Using $x+x^{\rho}+x^{\rho^{2}}=0$ and $y+y^{\rho}+y^{\rho^{2}}=0$, we get

$$
\begin{aligned}
v & =\left[x^{\rho}, y\right]-\left[x, y^{\rho}\right]=-\left[x^{\rho^{2}}, y\right]-[x, y]+\left[x, y^{\rho^{2}}\right]+[x, y]=\left[x, y^{\rho^{2}}\right]-\left[x^{\rho^{2}}, y\right] \\
v^{\rho} & =\left[x^{\rho^{2}}, y^{\rho}\right]-\left[x^{\rho}, y^{\rho^{2}}\right]=-\left[x^{\rho}, y^{\rho}\right]-\left[x, y^{\rho}\right]+\left[x^{\rho}, y^{\rho}\right]+\left[x^{\rho}, y\right]=\left[x^{\rho}, y\right]-\left[x, y^{\rho}\right]=v .
\end{aligned}
$$

Then

$$
v^{\sigma}=\left[x^{\rho \sigma}, y^{\sigma}\right]-\left[x^{\sigma}, y^{\rho \sigma}\right]=\left[x^{\rho^{2}}, y\right]-\left[x, y^{\rho^{2}}\right]=-v,
$$

hence $v \in H$ and, by triality, we have $v+v^{\rho}+v^{\rho^{2}}=3 v=0$. Since the characteristic of the field is not 3 then $v=0$ and we proved that

$$
\left[x^{\rho}, y\right]=\left[x, y^{\rho}\right], \quad\left[x^{\rho^{2}}, y^{\rho}\right]=\left[x^{\rho}, y^{\rho^{2}}\right] .
$$

Finally, we have by (2)

$$
\begin{aligned}
(x * y) * z & =\left[x+2 x^{\rho}, y\right] * z \\
& =\left[\left[x+2 x^{\rho}, y\right], z+2 z^{\rho}\right] \\
& =[[x, y], z]+2\left[\left[x^{\rho}, y\right], z\right]+2\left[\left[x+2 x^{\rho}, y\right], z^{\rho}\right] \\
& =[[x, y], z]+2\left[\left[x^{\rho}, y\right], z\right]+2\left[\left[x^{\rho}+2 x^{\rho^{2}}, y^{\rho}\right], z\right] \\
& =[[x, y], z]+2\left[\left[x^{\rho}, y\right], z\right]+2\left[\left[-x+x^{\rho^{2}}, y^{\rho}\right], z\right] \\
& =2\left[\left[x^{\rho^{2}}, y^{\rho}\right], z\right]+[[x, y], z] .
\end{aligned}
$$

Let $J=J(x, y, z)=(x * y) * z+(y * z) * x+(z * x) * y$, then by (1) we get

$$
J=2\left(\left[\left[x^{\rho^{2}}, y^{\rho}\right], z\right]+\left[\left[y^{\rho^{2}}, z^{\rho}\right], x\right]+\left[\left[z^{\rho^{2}}, x^{\rho}\right], y\right]\right) .
$$


But $t=\left[\left[x^{\rho^{2}}, y^{\rho}\right], z\right]-\left[\left[z^{\rho^{2}}, x^{\rho}\right], y\right]=0$, indeed, we have

$$
\begin{aligned}
t-t^{\rho}= & \left(\left[\left[x^{\rho^{2}}, y^{\rho}\right], z\right]-\left[\left[z^{\rho^{2}}, x^{\rho}\right], y\right]\right)^{\rho}-\left[\left[x^{\rho^{2}}, y^{\rho}\right], z\right]+\left[\left[z^{\rho^{2}}, x^{\rho}\right], y\right] \\
= & {\left[\left[x, y^{\rho^{2}}\right], z^{\rho}\right]-\left[\left[z, x^{\rho^{2}}\right], y^{\rho}\right]-\left[\left[x^{\rho^{2}}, y^{\rho}\right], z\right]+\left[\left[z^{\rho^{2}}, x^{\rho}\right], y\right] } \\
= & {\left[\left[x^{\rho^{2}}, y\right], z^{\rho}\right]-\left[\left[z, x^{\rho^{2}}\right], y^{\rho}\right]-\left[\left[x^{\rho^{2}}, y^{\rho}\right], z\right]+\left[\left[z^{\rho^{2}}, x^{\rho}\right], y\right] } \\
= & {\left[\left[x^{\rho^{2}}, z^{\rho}\right], y\right]+\left[x^{\rho^{2}},\left[y, z^{\rho}\right]\right]-\left[\left[z, y^{\rho}\right], x^{\rho^{2}}\right]-\left[z,\left[x^{\rho^{2}}, y^{\rho}\right]\right] } \\
& -\left[\left[x^{\rho^{2}}, y^{\rho}\right], z\right]+\left[\left[z^{\rho^{2}}, x^{\rho}\right], y\right] \\
= & {\left[x^{\rho^{2}},\left[y, z^{\rho}\right]\right]-\left[\left[z, y^{\rho}\right], x^{\rho^{2}}\right] } \\
= & 0 .
\end{aligned}
$$

Hence, $t \in H \cap\left\{v \mid v^{\rho}=v\right\}$. As above we can prove that $v=0$, since the characteristic of the field is $\neq 3$.

Then

$$
J(x, y, z)=(x * y) * z+(y * z) * x+(z * x) * y=6\left[\left[x^{\rho^{2}}, y^{\rho}\right], z\right] .
$$

Now we are ready to prove the Lemma. By (11) and (3) we get

$$
\begin{aligned}
& 2(x * y) * z+(z * y) * x+(x * z) * y \\
= & 3(x * y) * z+(z * y) * x+(x * z) * y+(y * x) * z \\
= & 3(x * y) * z+J(x, y, z) \\
= & 6\left[\left[x^{\rho^{2}}, y^{\rho}\right], z\right]+3[[x, y], z]-6\left[\left[x^{\rho^{2}}, y^{\rho}\right], z\right] \\
= & 3[[x, y], z],
\end{aligned}
$$

which proves the lemma.

Lemma 3.4. If a Lie algebra $L$ with triality is generated by elements $a_{1}, \ldots, a_{m}, a_{1}^{\alpha}, \ldots, a_{m}^{\alpha}$, where $a_{1}, \ldots, a_{m} \in H$, then the Malcev algebra $H$ is generated by $a_{1}, \ldots, a_{m}$.

Proof. We have $L=H \dot{+} S$, where $S=\left\{a \in L \mid a^{\sigma}=a\right\}$ and $H^{\alpha} \subseteq S$. Hence the subspace $H$ of $L$ is spanned by left-normed commutators $\left.b=\left[\ldots\left[b_{1}, b_{2}\right], b_{3}\right], \ldots, b_{r}\right]$, where $b_{1}, \ldots, b_{r} \in\left\{a_{1}, \ldots, a_{m}, a_{1}^{\alpha}, \ldots, a_{m}^{\alpha}\right\}$ and elements from $\left\{a_{1}, \ldots, a_{m}\right\}$ occur in $b$ an odd number of times.

1. Suppose that $b_{r}=a_{i}^{\alpha}, 1 \leq i \leq m, b^{\prime}=\left[\ldots\left[b_{1}, b_{2}\right], \ldots, b_{r-1}\right]$. Then by the induction assumption on $r$ the element $b^{\prime}$ lies in the Malcev algebra $H^{\prime}$ generated by $a_{1}, \ldots, a_{m}$ and $b=\left[b^{\prime}, a_{i}^{\alpha}\right]=-a_{i} * b^{\prime}$; 
2. Suppose that $b_{r} \in\left\{a_{1}, \ldots, a_{m}\right\}$. If the element $b_{r-1}$ also lies in $\left\{a_{1}, \ldots, a_{m}\right\}$ and $b^{\prime \prime}=\left[\ldots\left[b_{1}, \ldots, b_{r-2}\right]\right]$ then by the induction assumption $b^{\prime \prime} \in H^{\prime}$. In this case it remains to use Lemma 3.3 .

Let $b_{r-1} \in\left\{a_{1}^{\alpha}, \ldots, a_{m}^{\alpha}\right\}$. Then $b=\left[\ldots\left[b_{1}, \ldots,\left[b_{r-1}, b_{r}\right]\right]+\left[\ldots\left[b_{1}, \ldots, b_{r}\right], b_{r-1}\right]\right.$. By the induction assumption on $r$ applied to the elements $a_{1}, \ldots, a_{m}$, $a_{m+1}=\left[b_{r-1}, b_{r}\right] \in H^{\prime}$ the first summand lies in $H^{\prime}$. The second summand was considered in case 1 . This completes the proof of the lemma.

\section{Commutator identities in Groups}

Let $\operatorname{Fr}$ be the free group on free generators $x_{i}, i \geq 1 ; y, z_{1}, z_{2}$. Recall the Hall commutator identity

$$
[x y, z]=[y,[z, x]][x, z][y, z],
$$

where $[x, y]=x^{-1} y^{-1} x y$ is the group commutator.

Let $N$ be the normal subgroup of $F r$ generated by the element $y$ and let $N^{\prime}$ by the subgroup of $N$ generated by $[N, N]$ and by all elements $g^{p}, g \in N$. Then $N / N^{\prime}$ is a vectors space over the finite field $\mathbb{F}_{p}$. For an element $g \in F r$ consider the linear transformation

$$
g^{\prime}: N / N^{\prime} \rightarrow N / N^{\prime}, \quad h N^{\prime} \rightarrow[g, h] N^{\prime} .
$$

Then the Hall identity implies

$$
(a b)^{\prime}=a^{\prime}+b^{\prime}-b^{\prime} a^{\prime},
$$

or, equivalently, $1-(a b)^{\prime}=\left(1-b^{\prime}\right)\left(1-a^{\prime}\right)$, where 1 is the identity map. Hence, $1-\left(a^{p^{n}}\right)^{\prime}=\left(1-a^{\prime}\right)^{p^{n}}$. This implies the following well known lemma

Lemma 4.1. $\left.\left.[\underbrace{x_{1},\left[x_{1},\left[\ldots\left[x_{1}\right.\right.\right.}_{p^{n}}, y]\right] \ldots\right]=\left[x_{1}^{p^{n}}, y\right] \bmod N^{\prime}$.

Corollary. $\left[\left[x_{1}, z_{1}\right],\left[\left[x_{1}, z_{1}\right],\left[\ldots,\left[\left[x_{1}, z_{1}\right], y\right] \ldots\right]=\left[\left[x_{1}, z_{1}\right]^{p^{n}}, y\right] \bmod N^{\prime}\right.\right.$

Applying the so called "collection process" of G. Higman [11] (see also [22]) we linearize this equality in $x_{1}$.

Lemma 4.2. The product

$$
\prod_{\pi \in S_{p^{n}}}\left[\left[x_{\pi(1)}, z_{1}\right],\left[\left[x_{\pi(2)}, z_{1}\right],\left[\ldots,\left[\left[x_{\pi\left(p^{n}\right)}, z_{1}\right], y\right] \ldots\right]\right.\right.
$$


with an arbitrary order of factors lies in the subgroup generated by elements $\left[\left[x_{i+1} \cdots x_{i_{r}}, z_{1}\right]^{p^{n}}, y\right], 1 \leq i_{1}<\cdots<i_{r} \leq p^{n}$, and commutators $c$ in $y, z_{1}, x_{1}, \ldots, x_{p^{n}}$ such that

(1) c involves all elements $y, x_{1}, \ldots, x_{p^{n}}$,

(2) some element $y$ or $x_{j}, 1 \leq j \leq p^{n}$, occurs in c at least twice.

Consider again a group with triality $G$ and the Lie algebra with triality $L=L_{p}(G)=\sum_{i=1}^{\infty} L_{i}$. The subspace $H=\left\{a \in L \mid a^{\sigma}+a=0\right\}$ is graded, i.e. $H=\sum_{i=1}^{\infty} H_{i}, H_{i}=H \cap L_{i}$.

Lemma 4.3. Suppose that for an arbitrary element $g \in G$ we have $[g, \sigma]^{p^{n}}=1$. Then

(1) for an arbitrary homogeneous element $a \in H_{i}, i \geq 1$, we have $\operatorname{ad}(a)^{p^{n}}=0$

(2) for arbitrary homogeneous elements $a_{1}, \ldots, a_{p^{n}}$ from $H$ we have

$$
\sum_{\pi \in S_{p^{n}}} \operatorname{ad}\left(a_{\pi(1)}\right) \cdots \operatorname{ad}\left(a_{\pi\left(p^{n}\right)}\right)=0 .
$$

Proof. For a homogeneous element $a \in H_{i}$ there exists an element $g \in G_{i}$ such that $a=[g, \sigma] G_{i+1}$. Then $a^{\left[p^{n}\right]}=[g, \sigma]^{p^{n}} G_{p^{n} i+1}=0$. This implies $\operatorname{ad}(a)^{p^{n}}=\operatorname{ad}\left(a^{\left[p^{n}\right]}\right)=0$.

Let $a_{1}, \ldots, a_{p^{n}}$ be homogeneous elements from $H, a_{i}=\left[g_{i}, \sigma\right] G_{n(i)+1}$, $g_{i} \in G_{n(i)}, b=g^{\prime} G_{j+1}, g^{\prime} \in G_{j}$. Applying Lemma 4.2 to $x_{i}=g_{i}$, $z_{1}=\sigma, y=g^{\prime}$ we get the assertion (2).

Lemma 4.4. For an arbitrary element $a \in H$ we have $\left[a, a^{\rho}\right]=0$.

Proof. We have already mentioned that for an arbitrary element $g \in$ $[G, \sigma]$ we have $\left[g, g^{\rho}\right]=1$, see [8]. Hence, $\left[g, g^{\rho}\right]=0$ in $L(G)$.

Let $a_{i} \in H_{i}, a_{j} \in H_{j}$ be homogeneous elements. We need to show that $\left[a_{i}, a_{j}^{\rho}\right]+\left[a_{j}, a_{i}^{\rho}\right]=0$. There exist elements $g_{i} \in G_{i}, g_{j} \in G_{j}$ such that $a_{i}=\left[g_{i}, \sigma\right] G_{i+1}, a_{j}=\left[g_{j}, \sigma\right] G_{j+1}$. In the free group $\operatorname{Fr}$ consider the element

$$
X=\left[\left[x_{1}, z_{1}\right],\left[x_{2}, z_{1}\right]^{z_{2}}\right]\left[\left[x_{2}, z_{1}\right],\left[x_{1}, z_{1}\right]^{z_{2}}\right] .
$$


Applying the Hall identity and the Collection Process in the free group Fr we get

$\left[\left[x_{1} x_{2}, z_{1}\right],\left[x_{1} x_{2}, z_{1}\right]^{z_{2}}\right]=\left[\left[x_{1}, z_{1}\right],\left[x_{1}, z_{1}\right]^{z_{2}}\right]\left[\left[x_{2}, z_{1}\right],\left[x_{2}, z_{1}\right]^{z_{2}}\right] \cdot X \cdot c_{1} \cdots c_{r}$, where $c_{1}, \ldots, c_{r}$ are commutators in $x_{1}, x_{2}, z_{1}, z_{2}$; each of these commutators involved both elements $x_{1}, x_{2}$ and at least one of these elements occurs more than once.

Substitute $x_{1}=g_{i}, x_{2}=g_{j}, z_{1}=\sigma, z_{2}=\rho$. Then the equality above in the free group $F r$ implies $X \in G_{i+j+1}$. Hence $\left[a_{i}, a_{j}^{\rho}\right]+\left[a_{j}, a_{i}^{\rho}\right]=0$, which completes the proof of the lemma.

Example 4.5. Let $L$ be a nilpotent 3-dimensional Lie algebra with basis $a, b, c$ and multiplication $[a, b]=c,[a, c]=[b, c]=0$. The group $S_{3}$ acts on $L$ via $a^{\sigma}=-a, b^{\sigma}=a+b, c^{\sigma}=c, a^{\rho}=b, b^{\rho}=-a-b, c^{\rho}=c$. The straightforward computation shows that $L$ is a Lie algebra with triality and that $\left[a, a^{\rho}\right]=-c \neq 0$.

Lemma 4.6. (1) For an arbitrary element $a \in H$, arbitrary $k \geq 1$, we have $\operatorname{ad}\left(a^{\alpha}\right)^{p^{k}}=\operatorname{ad}(a)^{p^{k}}+2 \rho^{-1} \operatorname{ad}(a)^{p^{k}} \rho$;

(2) for arbitrary elements $a_{1}, \ldots, a_{p^{k}} \in H$ we have

$$
\begin{aligned}
& \sum_{\pi \in S_{p^{k}}} \operatorname{ad}\left(a_{\pi(1)}^{\alpha}\right) \cdots \operatorname{ad}\left(a_{\pi\left(p^{k}\right)}^{\alpha}\right) \\
= & \sum_{\pi \in S_{p^{k}}} \operatorname{ad}\left(a_{\pi(1)}\right) \cdots \operatorname{ad}\left(a_{\pi\left(p^{k}\right)}\right)+2 \rho^{-1} \sum_{\pi \in S_{p^{k}}} \operatorname{ad}\left(a_{\pi(1)}\right) \cdots \operatorname{ad}\left(a_{\pi\left(p^{k}\right)}\right) \rho .
\end{aligned}
$$

Proof. We only need to prove part (11). Part (2) is obtained from (1) by linearization. We have $a^{\alpha}=a+2 a^{\rho}$. By Lemma $4.4\left[a, a^{\rho}\right]=0$. Hence,

$$
\operatorname{ad}\left(a^{\alpha}\right)^{p^{k}}=\operatorname{ad}(a)^{p^{k}}+2^{p^{k}} \operatorname{ad}\left(a^{\rho}\right)^{p^{k}}=\operatorname{ad}(a)^{p^{k}}+2 \rho^{-1} \operatorname{ad}(a)^{p^{k}} \rho .
$$

This completes the proof of the lemma.

We remark that the proof of linearized Engel identity in [6] contains a gap that is filled in this paper.

For an element $a \in H$ let $\operatorname{ad}^{*}(a)$ denote the operator of multiplication by $a$ in the Malcev algebra, $\operatorname{ad}^{*}(a): h \rightarrow a * h, \operatorname{ad}^{*}(a)=\operatorname{ad}\left(a^{\alpha}\right)$.

Lemma 4.7. (1) For an arbitrary homogeneous element $a \in H_{i}$, $i \geq 1$, we have $\operatorname{ad}^{*}(a)^{p^{n}}=0$ 
(2) for arbitrary elements $a_{1}, \ldots, a_{p^{n}} \in H$ we have

$$
\sum_{\pi \in S_{p^{n}}} \operatorname{ad}^{*}\left(a_{\pi(1)}\right) \cdots \operatorname{ad}^{*}\left(a_{\pi\left(p^{n}\right)}\right)=0 .
$$

Proof. Assertion (11) follows from Lemma 4.3.(1) and Lemma 4.6.(1). Assertion (2) follows from Lemma 4.3,(2) and Lemma 4.6,(2).

\section{LoCAL NiLPotence In MALCEV ALGEBRAS}

Proposition 5.1. Let $M=M_{1}+M_{2}+\cdots$ be a finitely generated graded Malcev algebra over a field of characteristic $p \neq 2,3$, such that

(i) $\operatorname{ad}^{*}(a)^{p^{n}}=0$ for an arbitrary homogeneous element $a \in M$,

(ii) $\sum_{\pi \in S_{p^{n}}} \operatorname{ad}^{*}\left(a_{\pi(1)}\right) \cdots \operatorname{ad}^{*}\left(a_{\pi\left(p^{n}\right)}\right)=0$ for arbitrary $a_{1}, \ldots, a_{p^{n}} \in M$.

Then the Malcev algebra $M$ is nilpotent and finite dimensional.

If $I$ is an ideal of a Malcev algebra $M$ then $\widetilde{I}=I^{2}+I^{2} \cdot M$ is also an ideal of $M$. Consider the descending chain of ideals $M^{[0]}=M, M^{[i+1]}=\widetilde{M^{[i]}}$. We say that a Malcev algebra $M$ is solvable if $M^{[n]}=(0)$ for some $n \geq 1$.

Lemma 5.2 (V.T. Filippov, [4]). A finitely generated solvable Malcev algebra over a field of characteristic $>3$ is nilpotent if and only if each of its Lie homomorphic images is nilpotent.

Consider the free Malcev algebra $M(m)$ on $m$ free generators $x_{1}, \ldots, x_{m}$. As always $\mathbb{N}=\{1,2, \ldots\}$ is the set of positive integers. The algebra $M(m)$ is $\mathbb{N}^{m}$-graded via

$$
\operatorname{deg}\left(x_{i}\right)=\left(0,0, \ldots,{ }_{i}, 0, \ldots, 0\right), \quad 1 \leq i \leq m, \quad M(m)=\bigoplus_{\gamma \in \mathbb{N}^{m}} M(m)_{\gamma}
$$

Let $I$ be the ideal of $M(m)$ generated by elements $\underbrace{a(a(\cdots a b) \cdots)}_{p^{n}}$ and elements $\sum_{\pi \in S_{p^{n}}} a_{\pi(1)}\left(a_{\pi(2)}\left(\cdots\left(a_{\pi\left(p^{n}\right)} b\right) \cdots\right)\right.$, where $a, a_{1}, \ldots, a_{p^{n}}, b$ run over all homogeneous elements of $M(m)$. Let

$$
M\left(m, p^{n}\right)=M(m) / I
$$


Lemma 5.3. The algebra $M\left(m, p^{n}\right)^{2}$ is finitely generated.

Proof. E.N. Kuzmin (see [14]) showed that for an arbitrary Malcev algebra $M$ we have $M^{[3]} \subseteq M^{2} \cdot M^{2}$. By [20] every Lie homomorphic image of $M\left(m, p^{n}\right)$ is a nilpotent algebra. Hence by Lemma 5.2 of V.T. Filippov there exists $t \geq 1$ such that $M\left(m, p^{n}\right)^{t} \subseteq M\left(m, p^{n}\right)^{[3]} \subseteq$ $M\left(m, p^{n}\right)^{2} M\left(m, p^{n}\right)^{2}$. Since the algebra $M\left(m, p^{n}\right)$ is $\mathbb{N}^{m}$-graded it implies that $M\left(m, p^{n}\right)^{2}$ is generated by products of $x_{1}, \ldots, x_{m}$ of length $\ell, 2 \leq \ell \leq t-1$. This completes the proof of the lemma.

Recall that an algebra is said to be locally nilpotent if every finitely generated subalgebra is nilpotent.

Lemma 5.4. Let $M=M_{1}+M_{2}+\cdots$ be a graded Malcev algebra that satisfies the assumptions (ii), (iii) of the Proposition. Let I be an ideal of $M$ such that both $I$ and $M / I$ are locally nilpotent. Then the algebra $M$ is locally nilpotent.

Proof. Let $M^{\prime}$ be a subalgebra of $M$ generated by $m$ homogeneous elements. Then $M^{\prime}$ is a homomorphic image of the Malcev algebra $M\left(m, p^{n}\right)$. By Lemma 5.3 the algebra $\left(M^{\prime}\right)^{2}$ is finitely generated. Arguing by induction on $\operatorname{dim}\left(M^{\prime}+I / I\right)$ we can assume that the algebra $\left(M^{\prime}\right)^{2}$ is nilpotent. Hence $M^{\prime}$ is a solvable algebra. By [20] all Lie homomorphic images of the algebra $M^{\prime}$ are nilpotent. Hence by Lemma 5.2 the algebra $M^{\prime}$ is nilpotent, which completes the proof of the lemma.

Lemma 5.5. Let $M=M_{1}+M_{2}+\cdots$ be a graded Malcev algebra that satisfies the assumptions (ii), (iii) of the Proposition. Then $M$ contains a largest graded locally nilpotent ideal $\operatorname{Loc}(M)$ such that the factor algebra $M / \operatorname{Loc}(M)$ does not contain nonzero locally nilpotent ideals.

Remark. For Lie algebras this assertion was proved in [13], [18]

Proof. Let $I_{1}, I_{2}$ be graded locally nilpotent ideals of $M$. Since the factor algebra $I_{1}+I_{2} / I_{1} \cong I_{2} / I_{1} \cap I_{2}$ is locally nilpotent it follows from Lemma 5.4 that the algebra $I_{1}+I_{2}$ is locally nilpotent.

Let $\operatorname{Loc}(M)$ be the sum of all graded locally nilpotent ideals of $M$. We showed that the ideal $\operatorname{Loc}(M)$ is locally nilpotent. By Lemma 5.4 
the factor algebra $\bar{M}=M / \operatorname{Loc}(M)$ does not contain nonzero graded locally nilpotent ideals. Let $J$ be a nonzero (not necessarily graded) locally nilpotent ideal of $\bar{M}$. Let $J_{\mathrm{gr}}$ be the ideal of $\bar{M}$ generated by nonzero homogeneous components of elements of $J$ of maximal degree. It is easy to see that the ideal $J_{\mathrm{gr}}$ of $\bar{M}$ is locally nilpotent, a contradiction. This completes the proof of the lemma.

Recall that an algebra $A$ is called prime if for any nonzero ideals $I, J$ of $A$ we have $I J \neq(0)$. A graded algebra $A=A_{1}+A_{2}+\cdots$ is graded prime if for any nonzero graded ideals $I, J$ we have $I J \neq(0)$. Passing to ideals $I_{\mathrm{gr}}, J_{\mathrm{gr}}$ we see that a graded prime algebra is prime.

The proof of the following lemma follows a well known scheme (see [21]). We still include it for the sake of completeness.

Lemma 5.6. Let $M=M_{1}+M_{2}+\cdots$ be a graded Malcev algebra satisfying the assumptions (i), (ii) of the Proposition. Then the ideal $\operatorname{Loc}(M)$ is an intersection $\operatorname{Loc}(M)=\bigcap P$ of graded ideals $P \triangleleft M$ such that the factor algebra $M / P$ is prime.

Proof. Choose a homogeneous element $a \in M \backslash \operatorname{Loc}(M)$. Since the ideal $I(a)$ generated by the element $a$ in $M$ is not locally nilpotent there exists a finitely generated graded subalgebra $B \subseteq I(a)$ that is not nilpotent. Since the algebra $B$ satisfies the assumptions (i), (ii) it follows from V.T. Filippov's Lemma 5.2 that the algebra $B$ is not solvable.

Consider the descending chain of subalgebras $B^{(0)}=B, B^{(i+1)}=$ $\left(B^{(i)}\right)^{2}$. Since the algebra $B$ is not solvable we conclude that $B^{(i)} \neq(0)$ for all $i \geq 0$.

By Zorn's Lemma there exists a maximal graded ideal $P$ of $M$ with the property that $B^{(i)} \nsubseteq P$ for all $i$. Indeed, let $P_{1} \subseteq P_{2} \subseteq \cdots$ be an ascending chain of graded ideals such that $B$ is not solvable modulo each of them. If $B$ is solvable modulo $\bigcup_{i \geq 1} P_{i}$ then $B^{(s)} \subseteq \bigcup_{i \geq 1} P_{i}$ for some $s \geq 1$. By Lemma 5.3 the subalgebra $B^{(s)}$ is finitely generated, hence $B^{(s)} \subseteq P_{i}$ for some $i$, a contradiction.

We claim that the factor algebra $M / P$ is graded prime. Indeed, suppose that $I, J$ are graded ideals of $M, P \subsetneq I, P \subsetneq J$, and $I J \subseteq P$. 
By maximality of $P$ there exists $i \geq 1$ such that $B^{(i)} \subseteq I$ and $B^{(i)} \subseteq J$. Then $B^{(i+1)} \subseteq P$, a contradiction. This completes the proof of the lemma.

Proof of Proposition 5.1. Let $M$ be a graded Malcev algebra satisfying the assumptions (ii), (ii). If $M$ is not nilpotent then $M \neq \operatorname{Loc}(M)$. By Lemma 5.6, $M$ has a nonzero prime homomorphic image. V.T. Filippov [4] showed that every prime non-Lie Malcev algebra over a field of characteristic $p>3$ is 7-dimensional over its centroid. Now it remains to refer to the result of E.L. Stitzinger [19] on Engel's Theorem in the form of Jacobson for Malcev algebras. This completes the proof of the Proposition.

\section{Proof of Theorem 1}

Let $U\left(m, p^{n}\right)$ be the free Moufang loop of exponent $p^{n}$ on $m$ free generators $x_{1}, \ldots, x_{m}$. Let $E=E\left(U\left(m, p^{n}\right)\right)$ be the minimal group with triality that corresponds to the loop $U\left(m, p^{n}\right)$ (see [9]). The group $E$ is generated by elements $x_{1}, \ldots, x_{m}, x_{1}^{\rho}, \ldots, x_{m}^{\rho}$. Consider the Zassenhaus descending chain of subgroups $E=E_{1}>E_{2}>\cdots$. Let

$$
G=E / \bigcap_{i \geq 1} E_{i}, \quad U=[G, \sigma]
$$

Theorem 4 from [5] implies that an arbitrary finite $m$-generated Moufang loop of exponent $p^{n}$ is a homomorphic image of the loop $U$. We will show that the loop $U$ is finite.

As above, consider the Lie $p$-algebra $L=L_{p}(G)=\bigoplus_{i \geq 1} L_{i}, L_{i}=$ $G_{i} / G_{i+1}$, over the field $\mathbb{F}_{p},\left|\mathbb{F}_{p}\right|=p$, and the Malcev algebra $H=\left\{a-a^{\sigma} \mid a \in L\right\}$. The Malcev algebra $H$ is graded, $H=\bigoplus_{i \geq 1} H_{i}, H_{i}=$ $H \cap L_{i}$, and satisfies the assumptions (ii), (ii) of the Proposition.

Consider the Lie subalgebra $L^{\prime}$ of $L$ generated by the set $I_{m}=$ $\left\{a_{1}, \ldots, a_{m}, a_{1}^{\alpha}, \ldots, a_{m}^{\alpha}\right\}$, where $a_{i}=x_{i} E_{2} \in L_{1}, 1 \leq i \leq m$. The whole Lie algebra $L$ is generated by $I_{m}$ as a $p$-algebra.

Since the subalgebra $L^{\prime}$ is $S_{3}$-invariant it follows that $L^{\prime}$ is a Lie algebra with triality. Therefore $L^{\prime}$ gives rise to the Malcev algebra 
$H^{\prime}=L^{\prime} \cap H$. By Lemma 3.4 the elements $a_{1}, \ldots, a_{m}$ generate $H^{\prime}$ as a Malcev algebra. Hence, by Proposition 5.1 the algebra $H^{\prime}$ is nilpotent and finite dimensional. Let $\operatorname{dim}_{\mathbb{F}_{p}} H^{\prime}=d$.

Since the Lie algebra $L$ is generated by $a_{1}, \ldots, a_{m}$ as a $p$-algebra it follows that $L$ is spanned by $p$-powers $c^{\left[p^{k}\right]}$, where $c$ is a commutator in $a_{1}, \ldots, a_{m}$ of length $\leq 2 d, k \geq 0$. The space $H$ is spanned by $p$-th powers $c^{\left[p^{k}\right]}$, where the commutators $c$ have odd length.

An arbitrary homogeneous element $a \in H_{i}$ can be represented as $[g, \sigma] G_{i+1}$, where $g \in G_{i}$. Hence $[g, \sigma]^{p^{n}}=1$ implies $a^{\left[p^{n}\right]}=0$. Then $H$ is spanned by $p$-powers $c^{\left[p^{k}\right]}$, where $c$ is a commutator in $a_{1}, \ldots, a_{m}$ of odd length $\leq 2 d$ and $k<n$. Hence, $\operatorname{dim}_{\mathbb{F}_{p}} H<\infty$. Since $|H|=|U|$ we conclude that $|U|<\infty$. This concludes the proof of Theorem 1 .

\section{ACKNOWLEDGEMENTS}

The first author was supported by CNPq (grant 307824/2016-0), by FAPESP (Brazil) and Russian Foundation for Basic Research (under grant 16-01-00577a). The second author was supported in part by SNI-CONACyT, PRODEP-UAEM "Estancias cortas de investigacion de integrantes de Cuerpos Academicos Consolidados-2020", FAPESP 2019/24418-0 and UC MEXUS grants. The third author gratefully acknowledges the support of the NSF grant DMS 1601920.

\section{REFERENCES}

[1] Richard Hubert Bruck, A survey of binary systems, vol. 20, Springer, 1971.

[2] J. D. Dixon, M. P. F. du Sautoy, A. Mann, and D. Segal, Analytic pro-p groups, second ed., Cambridge Studies in Advanced Mathematics, vol. 61, Cambridge University Press, Cambridge, 1999.

[3] Stephen Doro, Simple Moufang loops, Math. Proc. Cambridge Philos. Soc. 83 (1978), no. 3, 377-392.

[4] V.T. Filippov, The Engel algebras of Malcev, Algebra and Logic 15 (1976), no. 1, 89-109.

[5] George Glauberman, On loops of odd order. II, J. Algebra 8 (1968), 393-414.

[6] A. N. Grishkov, The weakened Burnside problem for Moufang loops of prime period, Sibirsk. Mat. Zh. 28 (1987), no. 3, 60-65, 222.

[7] Alexander Grishkov, Lie algebras with triality, J. Algebra 266 (2003), no. $2,698-722$. 
[8] Alexander N. Grishkov and Andrei V. Zavarnitsine, Lagrange's theorem for Moufang loops, Math. Proc. Cambridge Philos. Soc. 139 (2005), no. 1, 41-57.

[9] Alexander N. Grishkov and Andrei V. Zavarnitsine, Groups with triality, J. Algebra Appl. 5 (2006), no. 4, 441-463.

[10] Alexander N. Grishkov and Andrei V. Zavarnitsine, Sylow's theorem for Moufang loops, J. Algebra 321 (2009), no. 7, 1813-1825.

[11] Graham Higman, Lie ring methods in the theory of

finite nilpotent groups, Proc. Internat. Congress Math. 1958, Cambridge Univ. Press, New York, 1960, pp. 307-312.

[12] Nathan Jacobson, Lie algebras, Interscience Tracts in Pure and Applied Mathematics, No. 10, Interscience Publishers, New York-London, 1962.

[13] A. I. Kostrikin, On Lie rings satisfying the Engel condition, Dokl. Akad. Nauk SSSR (N.S.) 108 (1956), 580-582.

[14] E. N. Kuzmin, Structure and representations of finite dimensional Malcev algebras, Quasigroups Related Systems 22 (2014), no. 1, 97-132.

[15] Gabor P. Nagy, Burnside problems for Moufang and Bol loops of small exponent, Acta Sci. Math. (Szeged) 67 (2001), no. 3-4, 687-696.

[16] Peter Plaumann and Liudmila Sabinina, On nuclearly nilpotent loops of finite exponent, Comm. Algebra 36 (2008), no. 4, 1346-1353.

[17] Peter Plaumann and Liudmila Sabinina, Some remarks on the Burnside problem for loops, Advances in algebra and combinatorics, World Sci. Publ., Hackensack, NJ, 2008, pp. 293-302.

[18] B. I. Plotkin, Algebraic sets of elements in groups and Lie algebras, Uspehi Mat. Nauk 13 (1958), no. 6 (84), 133-138.

[19] Ernest L. Stitzinger, On nilpotent and solvable Malcev algebras, Proc. Amer. Math. Soc. 92 (1984), no. 2, 157-163.

[20] E. I. Zelmanov, Solution of the restricted Burnside problem for groups of odd exponent, Izv. Akad. Nauk SSSR Ser. Mat. 54 (1990), no. 1, 42-59, 221.

[21] E. I. Zelmanov, Solution of the restricted Burnside problem for 2-groups, Mat. Sb. 182 (1991), no. 4, 568-592.

[22] Efim Zelmanov, Nil rings and periodic groups, KMS Lecture Notes in Mathematics, Korean Mathematical Society, Seoul, 1992.

[23] K. A. Zhevlakov, A. M. Slinko, I. P. Shestakov, and A. I. Shirshov, Rings that are nearly associative, Pure and Applied Mathematics, vol. 104, Academic Press, Inc., New York-London, 1982.

\footnotetext{
${ }^{A}$ Department of Mathematics, University of São Paulo, São Paulo, Brazil, and Omsk F.M. Dostoevsky State University, Omsk, Russia, E-mail address: grishkov@ime.usp.br;
}

${ }^{\mathrm{B}}$ Department of Mathematics, Autonomous University of the State of Morelos, Cuernavaca, Mexico,

E-mail address: liudmila@uaem.mx; 
${ }^{\mathrm{C}}$ Department of Mathematics, University of California, San Diego, USA E-mail address: ezelmanov@math.ucsd.edu

1. TO WHOM CORRESPONDENCE SHOULD BE ADDRESSED E-MAIL: LIUDMILA@UAEM.MX 